\title{
MODELO DIDÁCTICO TECNOSOCIAL: UNA EXPERIENCIA DE EDUCACIÓN PARA LA CIUDADANÍA CON JÓVENES UNIVERSITARIOS DESDE EL ESTUDIO DE LOS CONFLICTOS SOCIALES
}

\author{
Technosocial Didactic Model: \\ An Educational Experience for Citizenship with Young \\ University Students from the Study of Social Conflicts
}

\author{
Isabel Jiménez Becerra \\ isabel.jimenez@unisabana.edu.co \\ Universidad de La Sabana. Colombia
}

Fecha de recepción: 23/01/2020

Fecha de aceptación: 09/04/2020

RESUMEN: El artículo expone los resultados de una investigación orientada al rastreo, prototipo y testeo del modelo didáctico tecnosocial en la enseñanza de la Educación para la Ciudadanía EpC con jóvenes universitarios, a través del abordaje de los conflictos sociales. De esta manera se implementó un conjunto de iniciativas digitales para desarrollar el modelo, por medio de narrativas transmedia articuladas a escenarios tecnosociales. En el marco comprensivo sobre los conflictos sociales, el ejercicio de acción formativa examinó los alcances de cuatro categorías de análisis sobre el pensamiento histórico, a saber: juicio moral de la historia, contextualización, interpretación y empatía. Por consiguiente, se planteó un estudio de carácter cualitativo, con alcance descriptivo y un diseño de Investigación-Acción, para el trabajo con una muestra a conveniencia de 253 estudiantes, pertenecientes a ocho de los grupos dirigidos por la investigadora. Los resultados obtenidos evidencian un aporte al fortalecimiento del pensamiento crítico-creativo, la empatía social, la mirada sociocultural y la reflexión ciudadana como aspectos que influyen en la transformación de las problemáticas del contexto, a partir de la implementación de una secuencia didáctica.

Palabras clave: conflicto social; educación ciudadana; investigación pedagógica; pensamiento crítico; tecnología educativa. 
SUMMARY: The article exposes the results of an investigation oriented to the tracing, prototype and testing of a technosocial didactic model for the teaching of Education for Citizenship -EpCbased on social conflicts with university students. As a practical context for its teaching, digital initiatives were implemented to materialize the model through transmedia narratives articulated to technosocial settings. From the framework of the study of social conflicts, the formative action exercise examined the scope of four categories of analysis on historical thought: moral judgment of history, contextualization, interpretation and empathy. From a qualitative research, with a descriptive scope and an Action-Research design, we worked with a convenient sample of 253 students, belonging to eight of the groups led by the researcher. The results show a contribution to the development of critical-creative thinking, social empathy, cultural and ideological perspectives and the reflection of their actions as citizens to contribute to the transformation of contextual problems using for this a didactic sequence.

Keywords: social conflict; citizenship education; pedagogical research; critical thinking; educational technology.

SUMARIO: 1. Introducción. 2. Metodología. 3. Análisis de resultados. 3.1. Fases de planificación y acción: del juicio histórico a la contextualización de los conflictos sociales. 3.2. Fases de acción y reflexión: de la interpretación a la empatía de los conflictos sociales.4. Conclusiones. 5. Valoraciones finales. 6. Referencias bibliográficas.

\section{INTRODUCCIÓN}

Dentro del ámbito escolar, en el trabajo de identificar las prácticas de enseñanza de corte constructivista en Ciencias Sociales que permitan al estudiante reestructurar su imagen del mundo y las problemáticas de su entorno, implica considerar la historia como una herramienta de análisis, desde un ejercicio pedagógico situado en el plano de la empatía y el aprendizaje contextualizado (Santisteban, La formación de competencias de pensamiento histórico, 2010). De esta manera, se hace necesario socavar diferentes modelos didácticos y procesos de enseñanza a favor de la conciencia histórica y temporal, que se configuran desde las relaciones establecidas entre sus respectivas categorías: pasado, presente y futuro (Tutiaux-Guillon, 2003; Pagès, 2003; Pagès y Santisteban, 2008).

En este sentido, la memoria y los recuerdos generan una nueva concepción del presente y, por ende, son elementos que influyen en la cualificación y fortalecimiento de la educación ciudadana para la juventud actual, atendiendo a problemáticas reales. El análisis del contexto implica que los estudiantes replanteen los valores democráticos, implementando diversos ejercicios que los lleve a experimentar, de manera directa, la práctica de sus derechos y deberes. Como resultado, se proyecta una intervención social, donde comprender el presente hace parte de la construcción de un futuro mejor.

Un ejemplo tangible frente a la urgencia de replantear la enseñanza de las Ciencias Sociales, en relación con la Educación para la Ciudadanía (en adelante $\mathrm{EpC}$ ), se ubican en la memoria histórica colombiana donde la condición histórica 
del conflicto bélico tiene una trayectoria de medio siglo en la que ha cobrado la vida de más de 200000 personas. Por esta razón, varios países del mundo quedaron defraudados cuando la mayoría de los ciudadanos colombianos votaron por el NO a la implementación de los acuerdos de paz consensuados desde el 2016 entre las Fuerzas Armadas Revolucionarias (FARC) y el Gobierno.

Según datos presentados por la Registraduría Nacional del Estado Civil, el «NO» ganó con el 50,21 \% frente a un 49,78 \% que optó por el «Sí», con una tasa de abstención electoral que llegó al $63 \%$, en su mayoría jóvenes. Al respecto, Jiménez (2018) afirma que esto es producto de las prácticas pedagógicas orientadas hacia el reconocimiento del conflicto armado en el país, así como los valores democráticos centrados en el ejercicio al voto, traducido como la postura ciudadana frente a estas problemáticas sociales.

Al realizar un balance sobre la postura democrática centrada, bien sea en la abstención al voto o la elección del NO al paso del posconflicto, se identificaron opiniones en el marco de los juicios morales de la historia, mediatizados por las diversas representaciones sociales (Moscovici, 1988), identificada por otros como cuestiones sociales vivas (Tutiaux-Guillon, 2011). Sin duda, estas decisiones emanan de los prejuicios, estereotipos o imaginarios colectivos que emergen de los medios de comunicación (Santisteban, 2017), los hechos históricos en los libros textos, las narrativas de los profesores que enseñan las Ciencias Sociales o las experiencias de las personas víctimas del conflicto (Burke, 2006).

Entonces, surge el resentimiento y los sentimientos de victoria y derrota como resultado de la representación sobre los conflictos sociales y bélicos que poseen estos ciudadanos (Ferro, 2009). Estos aspectos han generado diversas preguntas dentro de la enseñanza del conflicto como estrategia $\mathrm{EpC}$, relacionadas con: ¿qué tipo de representaciones sociales poseen los alumnos sobre el conflicto? ¿Qué significado tiene la memoria histórica y el conflicto social, como estrategia didáctica en la enseñanza de las Ciencias Sociales? ¿Cuál es el papel de la educación frente al reconocimiento de las problemáticas de contexto, como base para educar en y para la ciudadanía?

Dentro de la búsqueda por responder efectivamente a estos interrogantes, en el año 2011 las Naciones Unidas realizó una investigación sobre el origen del conflicto armado y su efecto en la educación, donde se analiza la importancia de enseñar el conflicto como estrategia para la construcción de la paz (Unesco, 1998). No obstante, al revisar cómo desciende esta necesidad en las aulas, Méndez (2002, citado en Jiménez, 2018), analizó tanto el tipo de decisiones didácticas que asume el profesorado para administrar los contenidos curriculares, así como las estrategias metodológicas para la enseñanza de las Ciencias Sociales.

Allí Jiménez (2018) logró identificar las concepciones que poseían los profesores sobre el curriculum, como punto de encuentro entre las características de un modelo tradicional y una formación de tipo técnico. El riesgo de estas prácticas 
donde, además, se ha delegado al libro texto el poder de plantear los conceptos y contextos sobre la enseñanza para la construcción de ciudadanía, la educación cívica, la formación en valores y los derechos humanos, ha generado múltiples ambigüedades producto del uso de la metodología por casos alejadas de la realidad de quien enseña y de quien aprende. Es así como, en opinión de Jiménez (2018), la enseñanza del conflicto se reduce a la narración de hechos históricos, omitiendo toda posibilidad de interpretar los procesos de temporalidad y causalidad, para comprender el estado actual de la cuestión a partir de la contextualización de la realidad en sinergia con el análisis del hecho histórico estudiado.

Al profundizar las debilidades en las prácticas sobre la EpC, un factor alterno identificado ha sido la formación del profesorado en el marco de didáctica específica para la enseñanza. Allí Pagès (2002), citado por Jiménez (2018), sostiene que este tipo de didácticas se ocupan de conocer, analizar y valorar los problemas de la enseñanza y del aprendizaje de los conocimientos escolares para buscar soluciones, así como formar al profesorado para generar en ellos capacidades crítico-reflexivas para analizar y cualificar sus prácticas. Por consiguiente, se espera que el profesorado esté en la capacidad de pensar su práctica como un ejercicio didáctico donde confluye la reflexión, el aprendizaje, la interacción y el desarrollo del pensamiento crítico (Jiménez I. , Representación social del conflicto colombiano en los maestros de Básica Primaria , 2018).

Como resultado de esta problematización, se rastrearon modelos didácticos para la EpC que motivan a los jóvenes universitarios a ampliar su mirada cultural e ideológica sobre los conflictos sociales de su contexto. A partir de ahí, se buscó generar procesos centrados en la aceptación de la pluralidad, el conocimiento de sí y la toma de posición frente a diversas situaciones (Santisteban, La formación de competencias de pensamiento histórico, 2010); situación que, en palabras de Levesque (2008), citado por Santisteban (2010), implica el desarrollo de la imaginación, la empatía y la educación moral. Autores como Jiménez (2018) complementan el análisis al indicar que dichas categorías se logran cualificar en el aprendizaje producto del estudio contextualizado de la historia a partir de aspectos como los actores que intervinieron en ese hecho, sus motivaciones, la dinámica en juego siendo su interpretación la base del aprendizaje para comprender el presente y mejorar el futuro a partir de sus futuras acciones como ciudadano.

A nivel conceptual, los problemas sociales planteados por los jóvenes universitarios fueron relacionados con los deberes y derechos que vivencian como ciudadanos, mediante sus representaciones sociales y la interpretación de su papel dentro de su entorno. En consecuencia, fue necesario acudir a los mapeos realizados por Pagès y Santisteban (2014), quienes proponen una estructura conceptual en la que se enmarca la EpC. Esta estructura se materializó en una propuesta curricular que le permite al ciudadano tomar decisiones crítico-creativas, como un aporte a los procesos de empatía social y transformación de problemáticas de contexto. 
Los criterios aplicados para la elección de las teorías propuesta por Pagés y Santisteban (2014) son producto del anclaje del modelo conceptual y práctico en cualquier contexto mundial. La perspectiva de estos autores se orienta en cinco bloques articulados conceptualmente, a saber: la pluralidad y la organización social (integra conceptos como diferencia (diversidad/desigualdad), convivencia, organización (poder); la definición de ciudadanía (compuesta por elementos como libertad, igualdad, solidaridad, justicia, identidad y alteridad); los sistemas políticos: estructura y proceso político (aspectos como soberanía, sistemas de gobierno, estructura y proceso político); la cultura política como cultura democrática (con conceptos como socialización, ideología, cambio político, opinión pública, acción política). Finalmente, la cultura cívica para la intervención social (articula conceptos como racionalidad, responsabilidad, pensamiento crítico, resolución de conflictos).

Luego de este análisis, se planteó una investigación para viabilizar la respectiva trasposición pedagógica de este desafío educativo, centrada en generar una conciencia histórica sobre la realidad de los jóvenes universitarios. En este sentido, el camino de análisis se trazó desde las categorías de juicio moral de la historia, contextualización, empatía e interpretación, a través de las cuales se configura la pregunta sobre ¿Qué modelos de enseñanza implementa el profesorado para la comprensión e interpretación del contexto en sus estudiantes, como un aporte a la Educación para la Ciudadanía EpC?

Ante este panorama, se identificó una unidad didáctica creada por Pagès (1994) validada en el contexto español, como producto de diferentes investigaciones dirigidas e implementadas por el autor, en el marco de investigación rastreado por Jiménez (2018) en el Grup de Recerca en Didàctica de les Ciències Socials (GREDICS) de la Universidad Autónoma de Barcelona. Parte de este modelo fue analizado en la tesis doctoral realizada por Jiménez (2018), a partir de las representaciones que posee el profesorado el conflicto social en Colombia, así como el uso de estas problemáticas para su enseñanza. A partir de este antecedente, se incorpora el modelo de estudio de conflictos desarrollado desde la Educación para la Paz propuesto por Wehr (citado en Fisas, 1987) y Grasa (2000). Como producto de su propuesta se plantea una guía cartográfica del conflicto mediante los siguientes pasos:
a. Descripción general del conflicto.
b. Historia o antecedentes del conflicto.
c. Contexto del conflicto.
d. Partes implicadas o protagonistas.
e. Problemas en juego.
f. Dinámicas desarrolladas.
g. Alternativas para solucionar el problema.
h. Regulación potencial del conflicto. 
Es importante considerar un aspecto que robusteció la investigación, relacionado con el desafío de las Tecnologías de la Información y la Comunicación (TIC) ${ }^{1}$, como pieza clave para operacionalizar la acción comunicativa y la enseñanza de esta secuencia didáctica por medio una red social. Este componente, en palabras de Requena (1994), citado por Camero (2017), es aquel escenario donde «las personas están en continua interacción generando nuevos modelos de comunidad, para transmitir información, servicios, experiencias de enseñanza y/o ayuda a través de los diferentes niveles de la sociedad» (p. 44).

En este modelo de comunidad se ubican los escenarios tecnosociales, bajo características como la interactividad, el desarrollo de diversas formas de comunicación y el relacionamiento desde intereses, preocupaciones, desafíos o prácticas que se consolidan en el ciberespacio. Justamente es en este espacio donde las personas se relacionan de manera multidireccional y pasan a ser "WEB ACTORES» (Pisani y Piotet, 2013). Para Camarero (2017) en este perfil los individuos dejan de ser ciudadanos pasivos y se convierten en «protagonistas en el acto de comunicar y en la participaciónacción que aporta a transformar los problemas sociales, ambientales o educativos en los que están interesados abordar usando las redes sociales» (p. 221).

Para el caso de esta investigación, el proyecto tecnosocial reunió a un grupo de jóvenes universitarios interesados en educar para la ciudadanía, acudiendo a los conflictos sociales como centro de estudio, mediante el modelo de estudio de conflictos desarrollado desde la Educación para la Paz propuesto por Wehr (citado en Fisas, 1987) y Grasa (2000). Esta cartografía que, como recurso de enseñanza, posee una inteción de participación-acción formativa dentro de la «Comunidad tecnosocial de Educación para la Ciudadanía-EpC-a partir de los conflictos sociales con mediación TIC» se materializó en diversas narrativas transmedia. Scolari (2014), citado por Camero (2015), define este tipo de narrativas desde dos rasgos:

Por una parte, se trata de un relato que se cuenta a través de múltiples medios y plataformas. La narrativa comienza en un cómic, continúa en una serie televisiva de dibujos animados, se expande en forma de largometraje y termina (¿termina?) incorporando nuevas aventuras interactivas en los videojuegos [...].

${ }^{1}$ La reflexión del uso de las TIC en el escenario escolar hace parte de los aspectos a investigar en el macroproyecto de investigación fundado y dirigido por Isabel Jiménez Becerra titulado Didácticas Innovadoras mediadas TIC: retos a partir de la ecología del aprendizaje. Su objetivo central es teorizar las concepciones, desafíos, criterios de inclusión y alcances de algunos modelos de integración didáctica con mediación TIC, demostrando el nivel de innovación disruptiva, radical o incremental en la educación. Existen más de cincuenta tesis dirigidas en este grupo que ha impactado varios contextos geográficos colombianos. Los resultados de la creación e implementación del modelo hacen parte de una exploración inicial adscrita a este proyecto, donde se combinan tres componentes de formación en los jóvenes: el uso de las Competencias Básicas Digitales, la formación ciudadana y el estudio de los conflictos sociales para desarrollar habilidades de orden cognitivo, social, ético y político. 
También se caracterizan por otro componente: una parte de los receptores no se limita a consumir el producto cultural, sino que se embarca en la tarea de ampliar el mundo narrativo con nuevas piezas textuales (p. 72).

En este caso, los estudiantes implementaron prácticas de curaduría de herramientas TIC que les permitió filtrar aspectos para la construcción del recurso, tales como las herramientas TIC más apropiadas para la producción de la secuencia didáctica a modo de narrativa transmedia (Scolari, 2014). También, el diseño tecnoinstruccional (Coll y Moreno, 2008) sobre los Storyboards, entendidos como conjunto imágenes mostradas en secuencia, con el fin de previsualizar una animación gráfica o interactiva que, en este caso, presentó la cartografía del conflicto Finalmente, la red social más pertinente como escenarios de comunicación e interacción tecnosocial.

Los resultados obtenidos en la implementación de esta secuencia permitieron mapear y clasificar las representaciones sociales de los estudiantes universitarios sobre el conflicto social. Posteriormente, analizar los aportes al sentido brindado por los participantes a los acontecimientos históricos, desde la empatía y la contextualización como elementos esenciales en su formación ciudadana, donde, sin duda, adquiere un papel trascendental la mediación TIC como contexto de creación, comunicación e interacción.

\section{METOdOLOGÍA}

La investigación concentró su análisis en cuatro categorías del pensamiento críticocreativo para reforzar en los jóvenes universitarios, a saber: juicio moral de la historia, contextualización, empatía e interpretación. A partir de ahí, se planteó una metodología de corte cualitativo con alcance descriptivo, dinamizada desde el diseño de Investigación-Acción. El criterio de selección partió de la importancia de que el profesor investigador cuestione su enseñanza, como una oportunidad para innovar, renovar, poner a prueba sus creencias, con el propósito de fortalecer su práctica profesional.

En esta medida, la Investigación-Acción constituye un estudio sobre una situación social, con el fin de mejorar la calidad de la acción dentro de la misma. Esta característica, en el marco de la investigación, se tradujo en el propósito de desarrollar los niveles de interpretación y empatía con los estudiantes, sobre el análisis del conflicto y su papel como ciudadanos (Elliott, 1993). Así, en opinión de Kemmis (1998), la Investigación-Acción es un proceso político que implica cambios que afectan a las personas como, por ejemplo, el cambio de paradigma de los jóvenes universitarios frente a su actuar como ciudadanos, lo cual conlleva progresivamente a renovaciones más amplias.

Las técnicas e instrumentos de investigación utilizadas para la recolección de los datos se clasifican según las fases del diseño. En la planificación, se usó un cuestionario de entrada para mapear las representaciones sociales de los jóvenes universitarios. Posteriormente, en la fase de acción y observación, se utilizó como 
instrumento una encuesta de percepción que buscó evaluar, por parte de la población participante (virtual o presencialmente), la experiencia de enseñanza implementada por los jóvenes, a partir del uso del Storyboard como recurso que materializó la secuencia didáctica.

En el momento de reflexión, se implementó un grupo focal con estudiantes, que voluntariamente decidieron participar, posterior al ejercicio de implementación. Esta técnica se realizó con el objetivo de mapear los tipos de conflicto en los que está inmersa la población, así como los juicios históricos que poseen sobre los mismos. Para ello, los jóvenes universitarios crearon e implementaron tres preguntas: ¿qué tipos de conflictos sociales experimentan este contexto?, ¿quiénes son los responsables de este?, ¿se ha resuelto por vía pacífica o por medio de la violencia?

En cuanto a la muestra de estudio, elegida por conveniencia, estuvo conformada por doscientos cincuenta y tres (253) personas pertenecientes a ocho grupos de estudiantes universitarios de diversos programas académicos. Esta muestra hace parte de una población total de ochocientos (800) estudiantes que cursaron la asignatura de Competencia Básica Digital² en el corte de 2019-II. Para esto se consideró que el eje diferenciador a investigar dentro de la asignatura parte de la pregunta sobre ¿cómo un modelo didáctico tecnosocial, centrado en la conciencia histórica y el conflicto social colombianos, aporta a la Educación para la Ciudadanía EpC en los jóvenes universitarios? A partir de ahí, se ha concretado la cuestión al trasegar los siguientes objetivos:

- Mapear y clasificar las representaciones sociales que poseen los estudiantes universitarios sobre el conflicto social, como producto de los juicios morales que poseen de la historia.

- Plantear algunos modelos de integración didáctica con mediación TIC creados por los jóvenes universitarios, como aporte al desarrollo de la contextualización, la interpretación y la empatía como ciudadano.

- Analizar los aportes del modelo didáctico tecnosocial, centrado en la conciencia histórica y el conflicto social colombianos en la EpC en los jóvenes universitarios, desde la incorporación de experiencias de empatía histórica y social.

Con esto en mente, se plantearon unas fases de investigación acompañadas de unas categorías y un conjunto de técnicas e instrumentos para la recolección de datos, definidas en la Tabla 1.

${ }^{2}$ Los estudiantes descritos cursaron la clase transversal orientada por el Centro de Tecnologías para La Academia titulada Competencia Básica Digital (CBD). Esta competencia es definida por la Universidad de la Sabana aquella capacidad que poseen los estudiantes para afrontar de manera crítica y reflexiva situaciones académicas y sociales en un entorno digital. Esta asignatura posee una labor de formación social alterna, centrada en generar procesos de enseñanza donde los conceptos de Educación para la Ciudadanía sean analizados en el marco de los problemas sociales. Para ello se utilizó la teoría base generada por los investigadores Pagès y Santisteban (2014; 2016). 


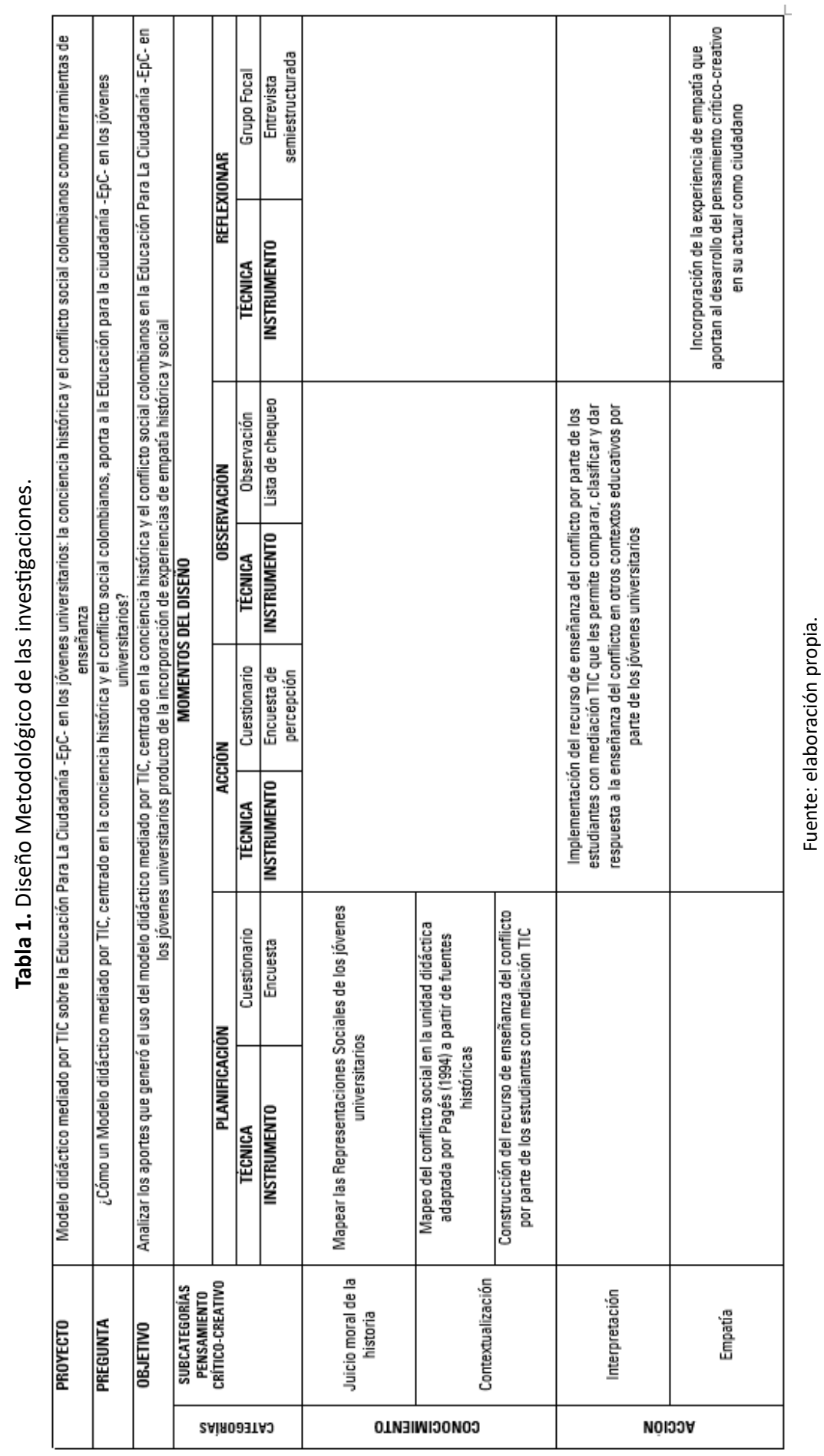


A continuación, se presentan los resultados de investigación clasificados desde las fases del diseño de Investigación-Acción, atendiendo a la relación establecida entre las categorías, subcategorías y los datos socavados al respecto.

\section{ANÁLISIS DE RESULTADOS}

Con la intención de dar respuesta a la pregunta de investigación, se implementó un ciclo de procedimientos del diseño de Investigación-Acción con una duración de un semestre. Estos ciclos de acción reflexiva (Lewin, 1946) o espirales de acción (Kemmis, 1998; McKernan, 1994; McNiff, 2014) permitieron extraer los resultados. De igual manera, analizar los aportes que generó el uso del modelo didáctico mediado por TIC, centrado en la conciencia histórica y el conflicto social colombianos en la EpC. Los resultados se reflejan en las diferentes fases:

\subsection{Fases de planificación y acción: del juicio histórico a la contextualización de los conflictos sociales}

La fase de planificación tuvo como objetivo mapear las representaciones sociales de los jóvenes universitarios participantes del estudio, así como identificar el conflicto social de su interés producto de los estereotipos, prejuicios e imaginarios históricos y sociales. Esto orientó la búsqueda de categorías de análisis, en el marco del pensamiento crítico creativo, para su actuar ciudadano y como parte de los juicios morales de la historia que poseen. De esta manera, se implementó el instrumento cuestionario para reconocer las representaciones sociales sobre conflicto de los jóvenes universitarios.

Este cuestionario estuvo conformado por seis (6) núcleos relacionados con creencia, ideología, percepción, actitud, estereotipo, que permitieron clasificar y codificar las diferentes representaciones sociales de los jóvenes universitarios sobre el conflicto social (Moscovici, 1988). El instrumento se interesó por la presentación y elección de diversas afirmaciones de las personas sobre lo que concibe, cómo percibe o cuál es su actitud frente a los conflictos sociales y el modo que los ubica dentro de un tipo de representación (Moscovici, 1988) y ajustadas por Jiménez (2018). En la Figura 1 se presentan los núcleos, sus principales preguntas y afirmaciones. 


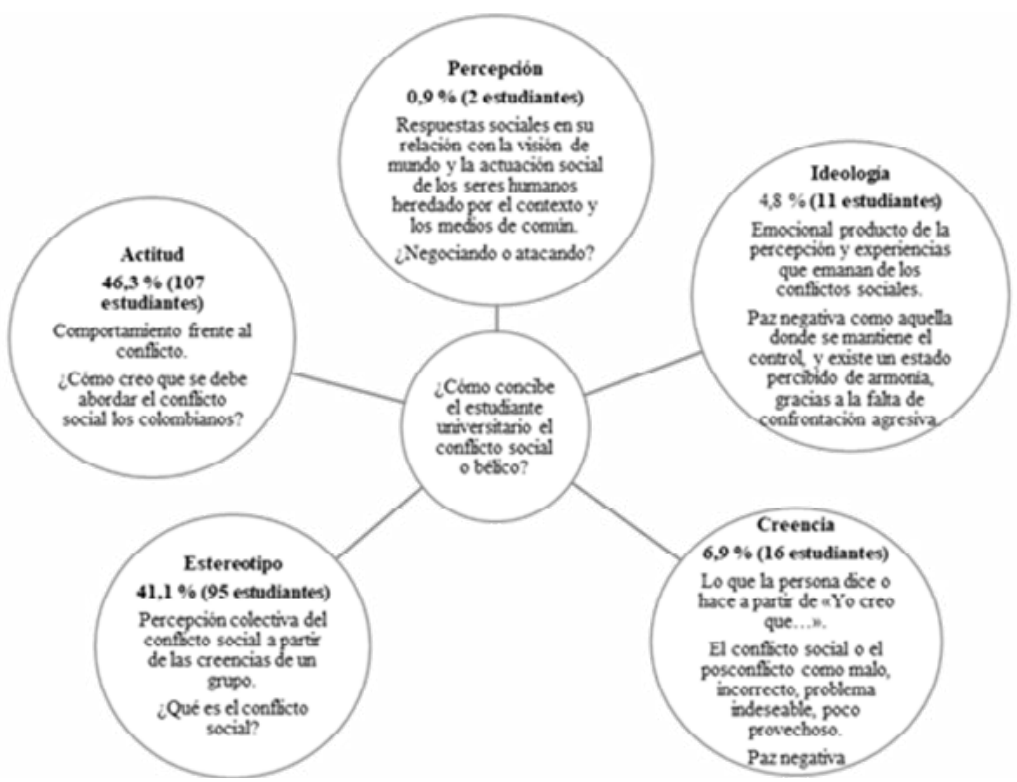

Figura 1. Representaciones sociales del conflicto que poseían los jóvenes universitarios. Fuente: Jiménez (2018).

Los resultados evidencian que el $41,1 \%$ de los estudiantes posee una representación social, producto del estereotipo construido sobre el conflicto bélico, como causa de la colisión social en el que está sumergido el país. Esta idea relacionada con la lucha que lleva el Estado contra los grupos revolucionarios, la falta de oportunidades, el desplazamiento, la firma de un acuerdo de paz, así como las pocas garantías de la persona para ejercer sus derechos fundamentales. Al preguntar por el origen de esta percepción, la mayoría de los jóvenes coincide que la información emerge de los escuchado en los medios de comunicación, escenario que les permite configurar una idea sobre el origen del conflicto (Santisteban, 2017).

Por otro lado, un 46,3 \% representan el conflicto social a partir de la actitud. Hubo una tendencia a relacionar las diferentes problemáticas sociales que experimenta el país, con la manera herrada como los ciudadanos han querido buscar la equidad y la justicia, acudiendo a la violencia y la guerra para lograr su objetivo. Diferentes respuestas coinciden en respaldar el proceso del proceso de paz, que inició en el 2012 en Colombia, como una entrada positiva al posconflicto. La mayoría de los participantes están convencidos que la negociación, la reparación, la justicia y el perdón son la vía para acabar con el conflicto armado del país.

De igual manera, el 6,9 \% de los jóvenes universitarios encuestados representan el conflicto desde las creencias. Se afirma que el conflicto social es algo malo, un problema que aqueja a la sociedad como resultado del comportamiento de los individuos, razón por la cual debe ser afrontado desde un marco legal contundente. Por su parte, el 4,8\% comprende el conflicto como una ideología, cuyo desenlace implica 
acabar con la confrontación social que existe como causa de diferentes problemáticas. Finalmente, un 0,9 \% de los participantes encuestados asume el conflicto social y bélico mediante la percepción, al sustentar que la guerra entre los diferentes grupos armados se ha controlado a través del uso de la fuerza e intervención del Estado. Consideran que este es el camino para terminar con las acciones violentas en el país.

Luego del mapeo que permitió clasificar representaciones sociales de los participantes sobre el conflicto social a partir de las estructuras propuesta por Jiménez (2018), que influyó en el análisis sobre su juicio moral de la historia, se dio paso a la segunda categoría del pensamiento crítico-creativo, conocida como contextualización del conflicto. En este momento, los estudiantes realizaron un proceso de exploración de los hechos históricos que han generado los conflictos sociales y bélicos actuales. Para esto utilizaron la unidad didáctica adaptada por Pagès (1994), a través del rastreo documental para reformular su conciencia histórica.

El uso de estrategias de enseñanza, como la graficación en mapas conceptuales, permitió que los estudiantes analizaran los caminos posibles para presentar su interpretación del conflicto elegido, junto con una reflexión sobre su papel como ciudadano en otros contextos educativos o corporativos. Para generar la trasposición de la secuencia didáctica Pagès (1994) en las narrativas transmedia (Scolari 2014), los estudiantes aplicaron prácticas de curaduría de herramientas TIC para la creación de productos como mapas mentales y conceptuales usando herramientas web, e-books, páginas web, cómics, podcasts, infografías, videos y redes sociales para socializar estos productos. Cada una de estas propuestas fue articulada a un portafolio digital, desde donde se avanzó en el proceso de investigación: plantear, teorizar, prototipar, testear y evaluar.

La actividad ejecutada con los estudiantes los llevó a utilizar los conocimientos históricos adquiridos en su formación, en contraste con lo rastreado y sus experiencias de vida. Dentro de este ejercicio, fueron replanteando sus representaciones del conflicto social, logrando así un despliegue reflexivo y conceptual de lo sucedido, lo que sucede y lo que sucederá. Por medio de técnicas como los cuestionarios y la observación, se planteó una encuesta de percepción y el levantamiento de evidencia fotográfica. Estos procesos son denominados por Santisteban (2010) como categorías de temporalidad humana, a partir de un análisis crítico que influye en el planteamiento de alternativas o soluciones a las nuevas problemáticas.

\subsection{Fases de acción y reflexión: de la interpretación a la empatía de los conflictos sociales}

La fase de acción tuvo como propósito orientar la implementación de las narrativas transmedia (Scolari, 2014) en contextos físicos o mediante el uso de redes sociales, dispuestas por los estudiantes para este ejercicio. La experiencia de Participación Acción les permitió comparar, clasificar y valorar el estudio del conflicto social desde la conciencia histórica. Desde ahí fue posible analizar la categoría 
interpretación, en cuanto proceso base para el desarrollo del pensamiento críticocreativo en su papel de ciudadanos (Pagès, 2003), como producto de las posturas de los participantes con quienes se implementaron las narrativas transmedia. Todo esto a partir de las fases del estudio de los conflictos propuesto en la Educación para la Paz de Wehr (citado en Fisas, 1987), Grasa (2000) y ajustada por Pagès (1994).

En cuanto al análisis de los datos obtenidos en la encuesta de caracterización, se identificó la participación de población perteneciente a cuatro contextos geográficos del departamento de Cundinamarca Colombia. Para esto se dividieron los doscientos cincuenta y tres (253) estudiantes en setenta y un (71) proyectos implementados, considerando los criterios de interés común, centrados en los conflictos sociales o bélicos que surgieron de sus representaciones sociales a priori. En la Figura 2 se exponen los datos estadísticos de los contextos y números de proyectos testeados en la implementación.

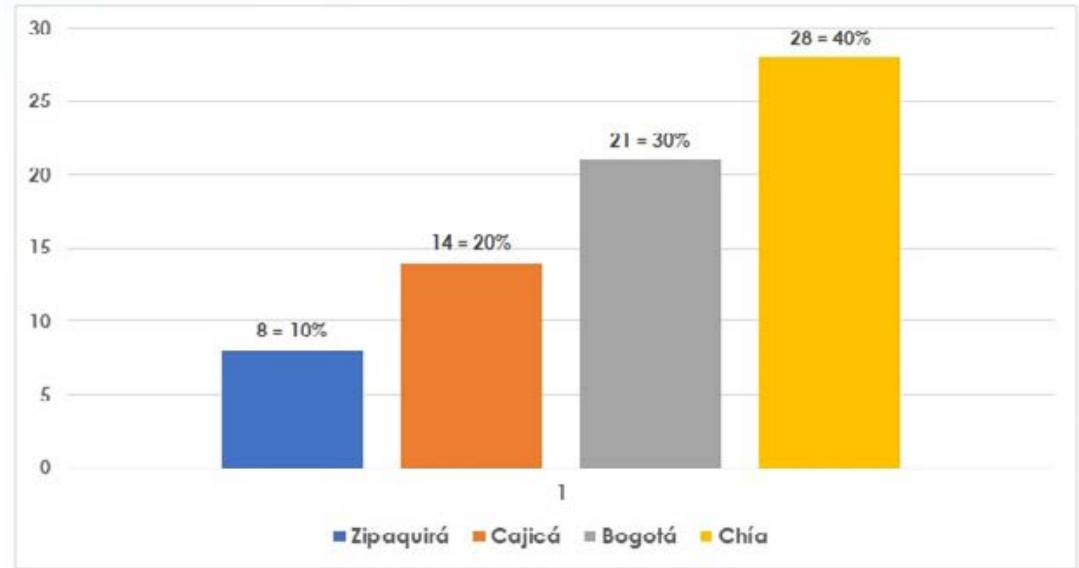

Figura 2. Zonas geográficas beneficiadas en la implementación del modelo didáctico. Fuente: elaboración propia.

La gráfica evidencia que el contexto con mayor impacto en el ejercicio de enseñanza fue Chía con un $40 \%$ (28 proyectos). En segundo lugar, Bogotá, con un $30 \%$ (21 proyectos), seguido de Cajicá, con un $20 \%$ (14 proyectos) y, finalmente, Zipaquirá, con un $10 \%$ (8 proyectos). Un segundo componente de la encuesta de percepción buscó evaluar, por parte de la población participante (virtual o presencialmente), la experiencia de enseñanza implementada por los participantes a partir del uso del Storyboard, como recurso que materializó la secuencia didáctica. Los investigadores lograron medir tres núcleos de análisis clasificados en: a) la percepción sobre la enseñanza los conflictos sociales, como aporte a la EpC; b) la evaluación de la actividad que otorgó un valor al uso del modelo didáctico creado; y c) el valor del recurso TIC para el aprendizaje. En la Figura 3, se muestran los resultados socavados que permitieron evaluar la percepción respecto al uso del material. 


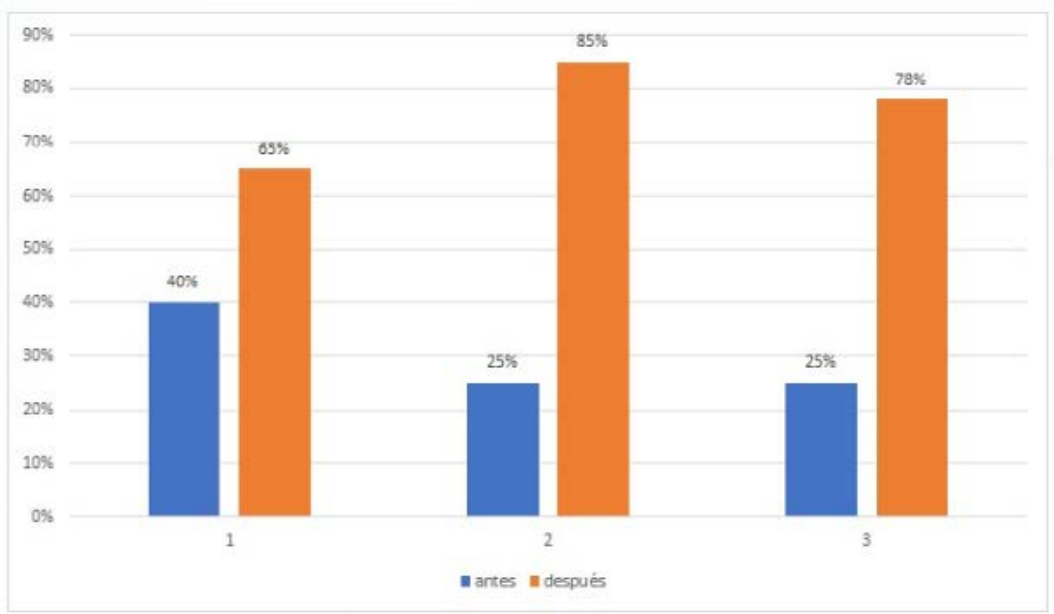

1. Percepción sobre la enseñanza de los conflictos sociales como aporte a la EpC

2. Evaluación de la actividad de intervención con mediación TIC que otorgó un valor al uso del modelo didáctico

3. El valor del recurso TIC para el aprendizaje

Figura 3. Nivel de percepción respecto al uso didáctico del modelo y su sinergia con la mediación TIC. Fuente: elaboración propia.

Al preguntar por la percepción de los participantes, el núcleo centrado en analizar el valor de la enseñanza de los conflictos sociales como aporte para EpC, fue evaluado por un $40 \%$ como favorable. Luego de experimentar el ejercicio de aprendizaje mediante el modelo didáctico propuesto, la percepción mejoró a un $80 \%$. Frente a la evaluación de la actividad de intervención, teniendo en cuenta la mediación y la apropiación de las herramientas tecnológicas, un $75 \%$ planteó el valor de su uso de la actividad compuesta por experiencias de gamificación. Esto se sustenta al comprender la importancia de los recursos TIC para el aprendizaje, pasando de un $40 \%$ a un $90 \%$ de satisfacción al experimentar con el ejercicio dispuesto.

Como cierre de la experiencia de investigación, en la fase metodológica titulada reflexión se buscó mapear cómo se logró incorporar experiencias de empatía que aportaron al desarrollo del pensamiento crítico-creativo en su actuar como ciudadano. Por esta razón, los estudiantes universitarios implementaron la técnica de los grupos focales con algunos voluntarios de la experiencia de implementación, a través de un cuestionario semiestructurado desde tres preguntas: ¿qué tipos de conflictos sociales experimentan este contexto?, ¿quiénes son los responsables de este?, ¿se ha resuelto por vía pacífica o por medio de la violencia?

A partir de las respuestas generadas por los participantes, los estudiantes lograron mapear los diversos conflictos sociales y bélicos en los que está inmersa 
estas poblaciones. Posteriormente, aplicaron de nuevo a la secuencia didáctica para la enseñanza del conflicto, a partir de estos nuevos conflictos declarados en las intervenciones del grupo focal. Las conclusiones de este ejercicio permitieron analizar cómo los participantes de la experiencia cambian paradigma de la representación del conflicto que, inicialmente, nace de los juicios históricos, aunque se transforman a partir de la interpretación contextualizada del mismo.

Sin duda, estos hechos se trasladan a las experiencias de empatía social al identificar los antecedentes históricos, los actores y la dinámica en juego, así como su papel de ciudadano para transformar tal problemática. Es así como, los resultados obtenidos en el grupo focal se organizan desde dos tipos de naturaleza, a saber:

a. Naturaleza social: el desplazamiento forzado, la pobreza, la desapropiación de tierras, la corrupción y la falta de equidad en salud y educación.

- Naturaleza bélica: relacionado con la migración de personas de otros contextos geográficos a las principales ciudades, como resultado del enfrentamiento de movimientos armados (guerrillas, paramilitarismos, grupos ilegales). También, con casos de violación de los derechos humanos descritos en masacres, asesinatos selectivos (líderes sociales) torturas, secuestros.

Por esta razón, los estudiantes universitarios mapearon los conflictos sociales y bélicos identificados, así como su localización en el marco de esta actual situación. De ahí que se acudiera a los análisis hechos por Gil (2018), quien retrata el tema del posconflicto desde el contexto de las víctimas, transformando sus juicios morales en saberes enmarcadas en el pensamiento histórico, mediante un análisis crítico reflexivo sobre sus implicaciones. Con este esquema, se han tomado las estadísticas sobre el total de víctimas que ha deja el conflicto bélico en Colombia. Además, los principales conflictos que han afectado a la población civil, siendo en su escala de menor a mayor: niños soldados, homicidios, torturas, víctimas de delitos sexuales, secuestrados, desapariciones y, el más latente, el desplazamiento (Figura 3). 


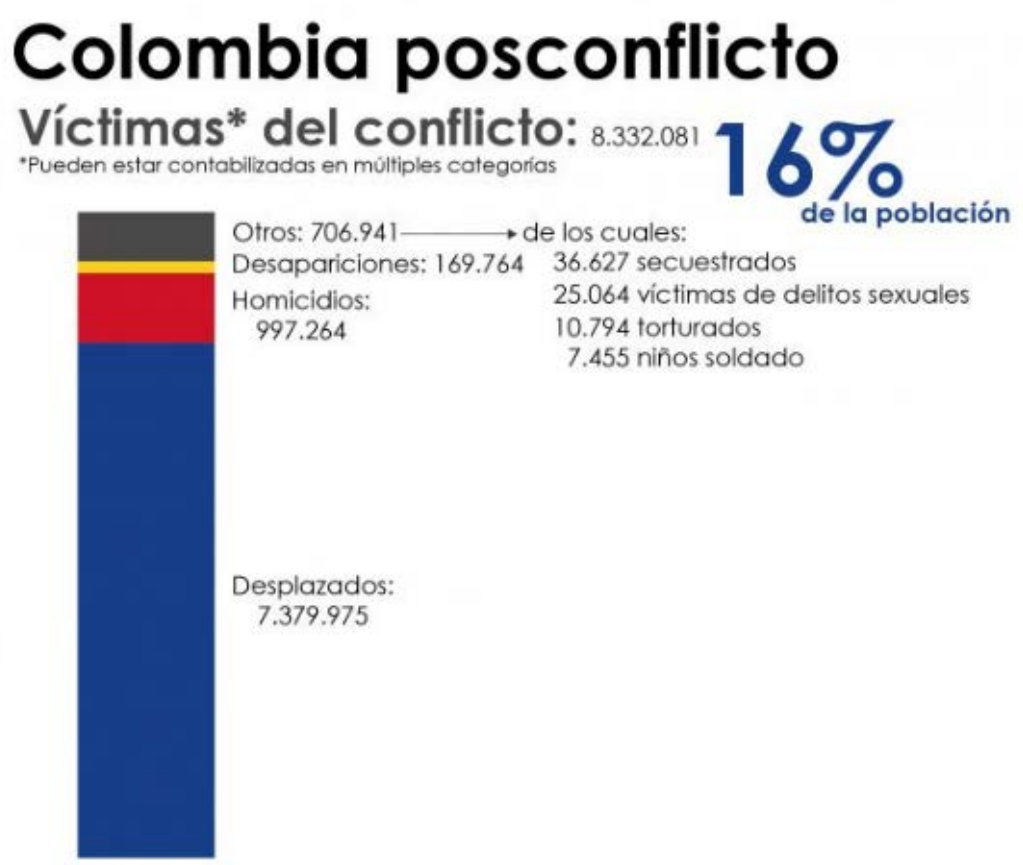

Figura 4. Datos de víctimas del conflicto en Colombia durante los últimos años. Fuente: adaptado de Gil (2018).

Posterior a esta radiografía, donde fue posible reconocer los diversos conflictos sociales y bélicos existentes en su entorno cercano, los estudiantes dieron un mayor sentido a categorías como la contextualización y la interpretación, derivados de la empatía que se puede generar de quien narra, de primera mano, las experiencias de conflicto como víctima. Al respecto, Barton y Levstik (2004) consideran las narrativas como poderosas herramientas culturales, dado que hacen parte de la cotidianidad del ser y, por ende, desarrollan un conjunto de habilidades para relatar actos de la vida humana; en este caso, tipos de conflicto frente a la experiencia de las víctimas (Amar, 2010).

\section{CONCLUSIONES}

Como conclusión de esta experiencia de Investigación-Acción, los jóvenes universitarios participantes replantearon el valor que tiene los hechos actuales por el que atraviesa Colombia, en el marco del posconflicto ${ }^{3}$. Desde aquí la educación

${ }^{3}$ Se entiende como el periodo de tiempo en el cual se implementan los acuerdos de paz, en cumplimiento del propósito de poner fin al conflicto armado y, por consiguiente, restablecer los derechos fundamentales de las víctimas. 
desempeña un papel vital en la comprensión y sostenibilidad de los acuerdos logrados, a partir del uso del diálogo como vía pacífica para su resolución.

Para mapear esta transformación en el pensamiento crítico creativo de los jóvenes universitarios, se acudió a recolectar las principales posturas de los participantes ubicadas en su portafolio digital, en una pestaña titulada autoevaluación (Márquez, Melo y Vergara, 2019). Dentro del marco de la investigación, estas autoevaluaciones fueron sintetizadas y clasificadas mediante las posturas de Moscovici (1986), como autor base de este estudio. En la Figura número 5 sobre la transformación del pensamiento crítico creativo de los participantes sobre los conflictos sociales y el efecto en su postura empática como ciudadano, se pueden encontrar los resultados correspondientes.

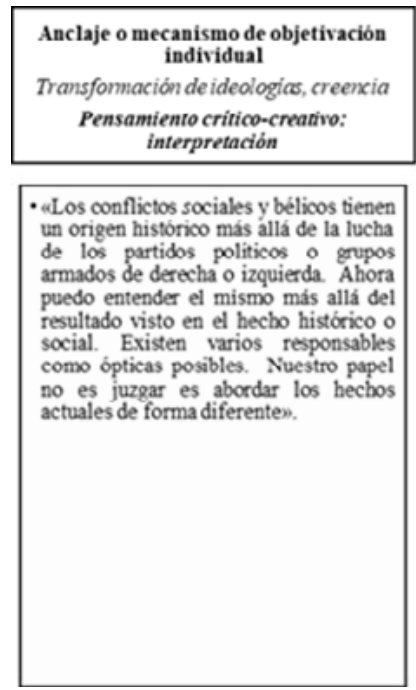

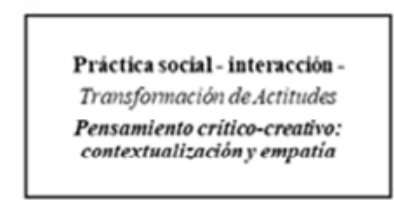

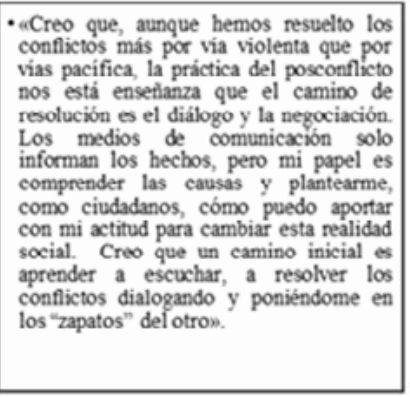

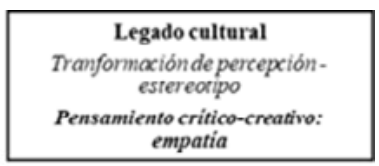

- $\alpha$ Los conflictos han sido resueltos por via violenta o pacifica $y$ eso no cambiara, pues hace parte de la memoria histórica estudiada. Lo que ahora debemos hacer para cambiar la resolución del conflicto por via violenta $y$ que hace parte de la experiencia del posconflicto, es trasegar por procesos de verdad (reconocer la historia, asi como los actores y sus acciones), pasando al nivel de justicia, que implica reconocer los emones, negociar los caminos de solución y reparar para dar paso asi al perdón... ahora entiendo que implica el posconflicto más allá de lo que escucho en las noticiasn.

Figura 5. Transformación del pensamiento- crítico creativo sobre los conflictos sociales. Fuente: elaboración propia.

Como fruto de estos ejercicios fue posible superar los juicios históricos que dominaban los jóvenes universitarios al inicio de esta investigación, presentes en la fase de la planificación. El ejercicio de identificar contextos de su interés para implementar el modelo didáctico, e interactuar con los participantes desde el mapeo de sus presaberes sobre el conflicto, implicó superar las valoraciones ingenuas producto de las representaciones sociales (Moscovici, 1988). A partir de ahí, orientarse hacia el reconocimiento de sus propias actitudes, creencias e intenciones en un determinado contexto histórico y cultural.

En cuanto a la evolución del pensamiento crítico-creativo, se logró avanzar hacia la cualificación de procesos de contextualización y empatía a partir de la 
interpretación de los hechos sociales e históricos, que motivó a los estudiantes a la comprensión de los problemas abordados como aporte a la transformación del entorno (Pagès y Santisteban, 2016), a partir de la implementación de acciones diferentes como ciudadano activo.

En opinión de Barton y Levstik (2004), luego de este tipo de experiencia, los estudiantes podrán participar en el reconocimiento de los problemas sociales y su papel como ciudadanos gestores de cambio. De manera que, tal y como lo plantea Santisteban (2010), el juicio moral a partir del estudio de la historia y los problemas de contexto constituye un componente importante de la enseñanza del pensamiento histórico en las escuelas; desde ahí, se presenta como una preparación para la vida democrática. En definitiva, la historia proyecta un cúmulo de creencias sobre la vida al mundo real, mediante el análisis de la propia acción humana, lo que se relaciona directamente con el pensamiento crítico.

Esto corroboró que la enseñanza, cuando se espera que el alumnado «viva la historia" y su experiencia histórica, puede ser un instrumento de motivación y de comprensión muy útil. De esta manera, el trabajo con fuentes, como las narrativas de los actores del conflicto bélico y social colombiano, donde según Santisteban (2010) deben implementarse desde andamiajes centrados en problemas históricos, donde el alumnado pueda desarrollar su competencia social histórica.

\section{VALORACIONES FINALES}

En opinión de Santisteban (2010), la formación de la conciencia histórica y su orientación temporal, desde el estudio del conflicto social o bélico propuesto en esta investigación, es fundamental para la formación democrática y ciudadanía. Es decir, se presenta como estrategia para fortalecer la convivencia y el desarrollo de habilidades sociales y ciudadanas, a favor de la participación democrática y la intervención social.

El estudio del conflicto, como parte de un interés de los jóvenes universitarios, reforzó la apropiación de categorías que conforman el pensamiento históricocreativo, transformando sus juicios morales y sus representaciones sociales sobre la historia, mediadas por los prejuicios y los estereotipos. Desde ahí, se generó una trasposición de las propias actitudes, creencias e intenciones en un determinado contexto histórico y cultural. En palabras de Barton y Levstik (2004), significa reconocer las problemáticas de contexto, mediante la contextualización, la interpretación y la empatía.

Una mirada alterna sobre el tema se encuentra en los postulados de Ennis (1985), quien relaciona el juicio moral en la clase de historia con la preparación para la vida democrática, como producto de las creencias obtenidas al experimentar un ciclo que va de la teoría a la experiencia. También, desde el análisis del mundo real, 
las razones de la acción humana y el papel del ciudadano, coherente con el desarrollo del pensamiento crítico.

Dentro de los aspectos a considerar en el estudio de las representaciones sociales, es superar el análisis de las percepciones, juicios, estereotipos o creencias de los jóvenes universitarios sobre el conflicto social y bélico actual. El uso del modelo didáctico también buscó incentivar las representaciones de las personas sobre el futuro. Al respecto, autores como Hicks \& Slaughter (1998) y Slaughter (1991), plantean cómo la reflexión de los problemas sociales, mediante estrategias como la memoria histórica, trazan futuros posibles, probables y deseables, teniendo en cuenta su accionar como ciudadano en el presente.

Por su parte, Santisteban (2010) recuerda que la implementación de prácticas de EpC, centrada en la formación de la conciencia histórica y su orientación temporal hacia el porvenir, es fundamental en la formación democrática de la ciudadanía. De este modo, la reflexión de los problemas sociales es la base para que los estudiantes evidencien una voluntad de transformación, así como una predisposición al compromiso a favor de los cambios sociales.

Finalmente, frente a la importancia del uso de las TIC para la enseñanza de las Ciencias Sociales como integración curricular tangible, aporta no solo al desarrollo de la competencia digital, también fomenta los aprendizajes significativos, motivadores y verdaderamente cooperativos colaborativos (Oller, 2011). Asimismo, promueve en el alumnado el desarrollo de habilidades cognitivas de orden superior, de diseñar y crear nuevos ambientes de aprendizaje o simulaciones virtuales que fomentan actitudes críticas y reflexivas sobre la información obtenida. De esta forma, se presentan como estrategias potentes para generar una sinergia entre los contenidos curriculares de la historia, las experiencias de enseñanza aprendizaje significativas que permitan el fortalecimiento de competencias de naturaleza social y cultural (Ortega, 2015).

Es de anotar que todas las secuencias didácticas creadas por los jóvenes universitarios para socializar la enseñanza y reflexión del conflicto social culminaron con una actividad de gamificación, en la búsqueda por potencializar la motivación, la concentración y el aprendizaje. De esta forma, se utilizaron herramientas web como Educaplay o Kahoot, teniendo en cuenta su potencial pedagógico y su contribución para flexibilizar los procesos pedagógicos, fortalecer habilidades cognitivas, espaciales y motoras, como fue demostrado por Jiménez y Escobar (2016). De igual manera, estas herramientas contribuyen al aprendizaje de hechos (conocimientos, memorización, repeticiones), principios (relación causa-efecto) y resolución de problemas complejos, aumentar la creatividad y aportar ejemplos prácticos de un concepto que es difícil ilustrar en el mundo real.

En este orden de ideas, Santisteban (2012, citado en Ortega, 2015) afirma que las TIC aportan a la comprensión de la realidad social, así como incentivar prácticas activas de ciudadanía a partir de estrategias didácticas y metodologías dirigidas 
competencias como la participación, toma de decisiones, la responsabilidad individual y grupal/colectiva. Como sucedió en el modelo didáctico propuesto por Wehr (citado en Fisas, 1987) y Grasa (2000), al cual se le articuló el uso de las TIC, se logró materialización las posturas del pensamiento crítico creativo aplicado por los estudiantes. Las estrategias articuladas a este modelo demuestran cómo pueden ser diseñadas propuestas de enseñanza a partir de situaciones problemas, donde el alumnado fundamente y exprese sus ideas a partir de las diversas situaciones reales analizadas por ellos. Finalmente, contrastar y dialogar con sus compañeros, en espacios de respeto, participación y formación ético-política, como uno de los fines pedagógicos y epistemológicos de las Ciencias Sociales.

\section{REFERENCIAS BIBLIOGRÁFICAS}

Amar, M. (2010). Instrucciones para la derrota: narrativas éticas y políticas de perdedores. Barcelona: Anthropos.

Barton, K., \& Levstik, L. (2004). Teaching History for the Common Good. Mahwah, New Jersey:Lawrence Erlbaum Associates. https://doi.org/10.4324/9781410610508.

Burke, P. (2006). ¿Qué es la historia cultural? Barcelona: Paidós.

Castells, M. (2003). L'era de la informació. Economia, societat i cultura. Vol. II: EI poder de la identitat. Vol. III: Canvi de milleni. Barcelona: Editorial UOC.

Elliott, J. (1993). El cambio educativo desde la investigación acción. Madrid: Morata.

Ennis, R. (1985). A logical basis for measuring critical thinking skills. Educational Leadership, 43(2), pp. 45-48.

Ferro, M. (2009). El Resentimiento en la historia. Madrid: Cátedra.

Fisas, V. (1987). Introducció a l'estudi de la pau i dels conflictes. Barcelona: Fundació Jaume Bofill.

Gómez, J., \& Torrijo, R. (2019). Problemática de la deforestación en la Amazonia Colombiana (hecho histórico conflicto Colombo-Peruano) . Obtenido de LiveBinders: https://www.livebinders.com/play/play?id=2536353\#anchor.

Grasa, R. (2000). Evolución de la Educación para la Paz La centralidad del conflicto para la agenda del siglo XXI. Revista Cuadernos de Pedagogía, 52-56. 
Hicks, D., \& Slaughter, R. (1998). Futures Education: World Yearbook of Education. London: Kogan page.

Jiménez, E. (2019, 28 de Marzo). Colombia, la paz que nunca llega. Obtenido de Lapipa.com: https://www.lapipa.co/2019/03/28/colombia-la-paz-que-nunca-llega/.

Jiménez, I. (2018). Representación social del conflicto colombiano en los maestros de Básica Primaria. Barcelona: Universidad Autónoma de Barcelona.

Jiménez, I., \& Escobar, C. (2016). Uso didáctico del videojuego educativo para la enseñanza de las Ciencias Sociales: un estado del arte. Paideia, 58, pp. 53-70.

Kemmis, S. (1998). El curriculum más allá de la teoría de la reproducción. Madrid: Morata.

Lévusque, S. (2008). Thinking Historically: Educating Students for the Twenty-first Century. Toronto: University of Toronto Press.

Lewin, K. (1946). Action Research and Minority Problems. Journal of Social Issues, 34-46. https://doi.org/10.1111/j.1540-4560.1946.tb02295.x.

Márquez, S., Melo, B., \& Vergara, J. (2019). Educación Especial para la Paz. Víctima: entre el conflicto armado. Obtenido de Canva: https://www.canva.com/design/ DADV2X2_BfE/nTq9OIn84J0bn7DiitxajA/view?utm_content=DADV2X2_BfE\&utm_ campaign=designshare\&utm_medium=link\&utm_source=sharebutton\#24.

McKernan, J. (1994). Investigación-acción y curriculum, métodos y recursos para profesionales reflexivos. Madrid: Morata.

McNiff, J. (2014). Writing and Doing Action Research. London: Sage Publications. https://doi.org/10.4324/9781315738499.

Moscovici, S. (1988). Les representations sociales. Expose introductif. En L. Marbeau y F. Audigier (Eds.), Seconde Rencontre Nationale sur la Didactique de l'Histoire et de la Géographie. París: Actes du colloque, INRP.

Oller, M. (2011). Métodos y estrategias para la enseñanza y el aprendizaje de las Ciencias Sociales. En A. Santisteban y J. Pagès (Coords.), Didáctica del Conocimiento del Medio Social y Cultural en la Educación Primaria: ciencias sociales para aprender, pensar y actuar. (pp. 163-184). Madrid: Síntesis. 
Ortega, D. (2015). a enseñanza de las Ciencias Sociales, las TIC y el Tratamiento de la Información y Competencia Digital (TICD) en el grado de maestro/a de Educación Primaria de las Universidades De Castilla y León. Enseñanza de las Ciencias Sociales, 14, pp. 121-135.

Pagès, J. (1994). Los conflictos en las sociedades rurales: el conflicto remensa (s. XV) y el conflicto de Chiapas. En P. Benejam, \& J. Pagès, Ciencias Sociales. Contenidos, actividades y recursos. Guías práxis para el profesorado de ESO (pp. 415-480). Barcelona: Práxis.

Pagès, J. (2002). Aprender a enseñar historia y Ciencias Sociales: el currículo y la didáctica de las Ciencias Sociales. Pensamiento educativo, 30(1), pp. 255-269.

Pagès, J. (2003). Ciudadanía y enseñanza de la historia. Reseñas de Enseñanza de la Historia, 1.

Pagès, J., \& Santisteban, A. (2008). Cambios y continuidades: aprender la temporalidad histórica. En M. Jara, Enseñanza de la Historia. Debates y propuestas. Neuquén.

Pagès, J., \& Santisteban, A. (2014). Una mirada del pasado al futuro en la didáctica de las Ciencias Sociales. Barcelona: Servicio de Publicaciones de la UAB.

Santisteban, A. (2010). La formación de competencias de pensamiento histórico. Memoria académica, 14, pp. 34-56. http://www.memoria.fahce.unlp.edu.ar/art_ revistas/pr.4019/pr.4019.pdf. https://doi.org/10.14409/cya.v1i14.1674.

Santisteban, A., \& Pagès, J. (2016). La enseñanza de la historia en la educación primaria. En M. Casas, \& C. Tomás, Educación Primaria. Orientaciones y Recursos (pp. 129-468). Barcelona: Wolters Kluwer Educación.

Slaughter, R. (1991). Futuros. En D. Hicks, Educación para la paz. Morata: Madrid.

Tutiaux-Guillon, N. (2003). L'histoire enseignee entre coutume disciplinaire et formation de la conscience historique: l'exemple français. En N. Tutiaux-Guillon, \& C. Nourrisson, Identités, mémoires, conscience historique. París: Université de Saint-É.

Unesco. (1998). Informe mundial sobre la educación: los docentes y la enseñanza en un mundo en mutación. París: Unesco. 